\title{
深俯冲大陆地壳的部分惨融：大别造山带超高压榴辉岩 中多相固体包裹体证据
}

\author{
高晓英"，郑永飞，陈伊翔 \\ 中国科学院壳幔物质与环境重点实验室, 中国科学技术大学地球和空间科学学院, 合肥 230026 \\ * 联系人, E-mail: gaoying@ustc.edu.cn \\ 2013-05-29 收稿, 2013-06-19 接受, 2013-07-29 网络版发表 \\ 国家重点基础研究发展计划(2009CB825004)和国家自然科学基金(41221062)资助
}

\begin{abstract}
摘要 在大别造山带双河地区中温超高压榴辉岩中, 石榴石含有不同类型的多相固体包裹 体, 从纯石英到纯钾长石, 以及钾长石十石英土其他硅酸盐(钠长石、斜长石、绿穷石、褐侘石 或重晶石)。这些多相固体包裹体以钾长石十石英为主, 呈细晶交互生长结构, 为自形至半自 形且具有放射状爆裂纹, 与石榴石或绿辉石中柯石英假象或柯石英包裹体类似。研究指示, 在深俯冲陆壳折返早期, 超高压岩石发生降压脱水乃至部分熔融, 形成了这些富 Si-K-Al 的 长英质熔体. 采用原位 LA-ICPMS 分析技术, 对长英质多相固体包裹体进行微量元素分析, 分别采用外剥法和内剥法这两种方法, 定量计算熔体微量元素组成. 结果显示, 两种方法给 出了相似的微量元素组成, 具有相对富集 LILE, Sr 和 $\mathrm{Pb}$, 相对亏损 HFSE 的微量元素分布型 式, 与弧型岩浆岩的分布型式相近. 熔体具有变化的 $\mathrm{K}, \mathrm{Rb}$ 和 $\mathrm{Sr}$ 含量, 说明可能是多硅白云 母脱水引发部分熔融, 继而形成具有长英质成分的多相固体包裹体.
\end{abstract}

\section{关键词}

多相固体包裹体

长英质熔体

部分熔融

大陆深俯冲

超高压变质
研究大陆碰撞带超高压变质岩所记录的部分熔 融现象, 对于深人理解大陆俯冲带矿物反应和元素 迁移行为、造山带热演化历史和折返动力学机制具有 重要意义 ${ }^{[1]}$. 因此, 确定碰撞造山带深熔作用发生的 岩石类型、空间分布、熔融机制和熔融时间是理解大 陆动力学的关键. 大规模的地壳深熔作用容易在露 头尺度上识别，一般以混合岩的形式出现. 小程度部 分熔融有可能只发生在薄片尺度, 只能通过显微结 构分析加以判别 ${ }^{[2]}$. 大别-苏鲁超高压变质带中广泛 出露经过超高压变质作用的各类岩石 ${ }^{[3]}$, 包括长英质 岩石类型的片麻岩和石英岩以及镁铁质岩石类型的 榴辉岩. 由于长英质岩石中可能含有长石类易熔组 分, 相对于镁铁质岩石来说更容易发生部分熔融, 因 此是研究大陆碰撞过程中超高压岩石发生深熔作用 的理想对象. 镁铁质岩石, 特别是榴辉岩, 可能只在
深俯冲大陆板片内部发生小规模深熔作用, 只能借 助现代岩相学显微研究.

大陆俯冲带超高压变质矿物中多相固体包裹体 作为熔体活动的直接记录, 为我们提供了揭示超高 压变质过程中部分熔融和熔体演化的重要对象 ${ }^{[4]}$. 近 年来, 围绕大别-苏鲁造山带超高压岩石中多相固体 包裹体的形成时间、演化过程及其所反映的大陆俯冲 带超高压变质成因熔体的组成和性质, 也已进行了 大量的研究工作 ${ }^{[5 \sim 12]}$. 超高压岩石中多相固体包裹体 的发现, 为理解峰期超高压变质流体的组成和演化 提供了重要制约, 同时也为研究俯冲板片-地幔楔界 面的熔体交代作用提供了新的途径 ${ }^{[1]}$. 本文以大别造 山带超高压变质榴辉岩为例, 从多相固体包裹体的 结构形态特征、矿物化学成分、地球化学特征及其包 裹体的形成机制等方面，对于超高压变质岩中多相 
固体包裹体进行了概括, 以期促进多相固体包裹体 的岩石学和地球化学研究.

\section{1 多相固体包裹体的岩相学特征}

多相固体包裹体, 即以两种或两种以上的固体 矿物集合体的形式出现的固体包裹体, 在形成初期 常含有一定的熔/流体相成分. 多相固体包裹体的寄 主矿物指用来包裹固体包裹体的矿物. 在超高压变 质环境中, 寄主矿物主要是超高压矿物, 如石榴石、 绿辉石、绿帘石和蓝晶石等. 这些包裹体在寄主矿物 的核部或边部均可分布, 自形程度依封闭于其中的 熔/流体情况而异, 大部分包裹体的大小 $<25 \mu \mathrm{m}$, 个 别可达到 $250 \mu \mathrm{m}$ 以上. 在理想的结晶环境下, 多相 固体包裹体可以整体表现出与寄主矿物相一致的晶 体形态一负晶形, 这是多相固体包裹体具有熔/流体 成因的最直接证据. 不过, 并非所有多相固体包裹体 都结晶得那么理想, 而且负晶形常会因多相固体包 裹体后期的退变演化而被破坏.

前人对双河榴辉岩的岩石学、同位素年代学和地

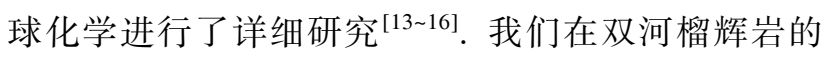
石榴石和绿辉石中发现了不同类型的固体多相包裹
体, 从纯石英到纯长石 (钾长石或钠长石), 以及长石 +石英土其他硅酸盐，包括有钠长石、斜长石、绿帘石、 褐帘石或重晶石(图 1)。这些多相固体包裹体以不同 类型的长石+石英组合为主, 呈细晶交互生长结构, 为自形至半自形且具有放射状懪裂纹, 与石榴石或 绿辉石中柯石英假象或柯石英包裹体类似.

采用电子探针和能谱分析, 对包裹体中的重晶石 进行矿物化学成分测定, 结果显示重晶石在主量元素 成分上存在着很大的不均一性，总量仅有 $57 \mathrm{wt} \%$ $73 \mathrm{wt} \%$, 远低于 $100 \mathrm{wt} \%$; 重晶石中 $\mathrm{SiO}_{2}$ 的含量具有 较大的波动范围, 变化从 $0.32 \mathrm{wt} \% \sim 25.85 \mathrm{wt} \%$, 并且 与 $\mathrm{SO}_{3}$ 和 $\mathrm{BaO}$ 之间呈很好的负相关性, 而在 $\mathrm{BaO}$ 与 $\mathrm{SO}_{3}$ 之间却呈现很好的正相关性(图 2). 这些结果表 明, 重晶石集合体包含微孔隙, 可能与石英呈微晶集 合体分布, 指示它们可能是同时从同一熔/流体相中 结晶出来的.

\section{2 多相固体包裹体的微量元素地球化学 特征}

在超高压变质岩中发现的多相固体包裹体, 其 大小通常 $<25 \mu \mathrm{m}$ ，大部分都小于 LA-ICPMS 的激光
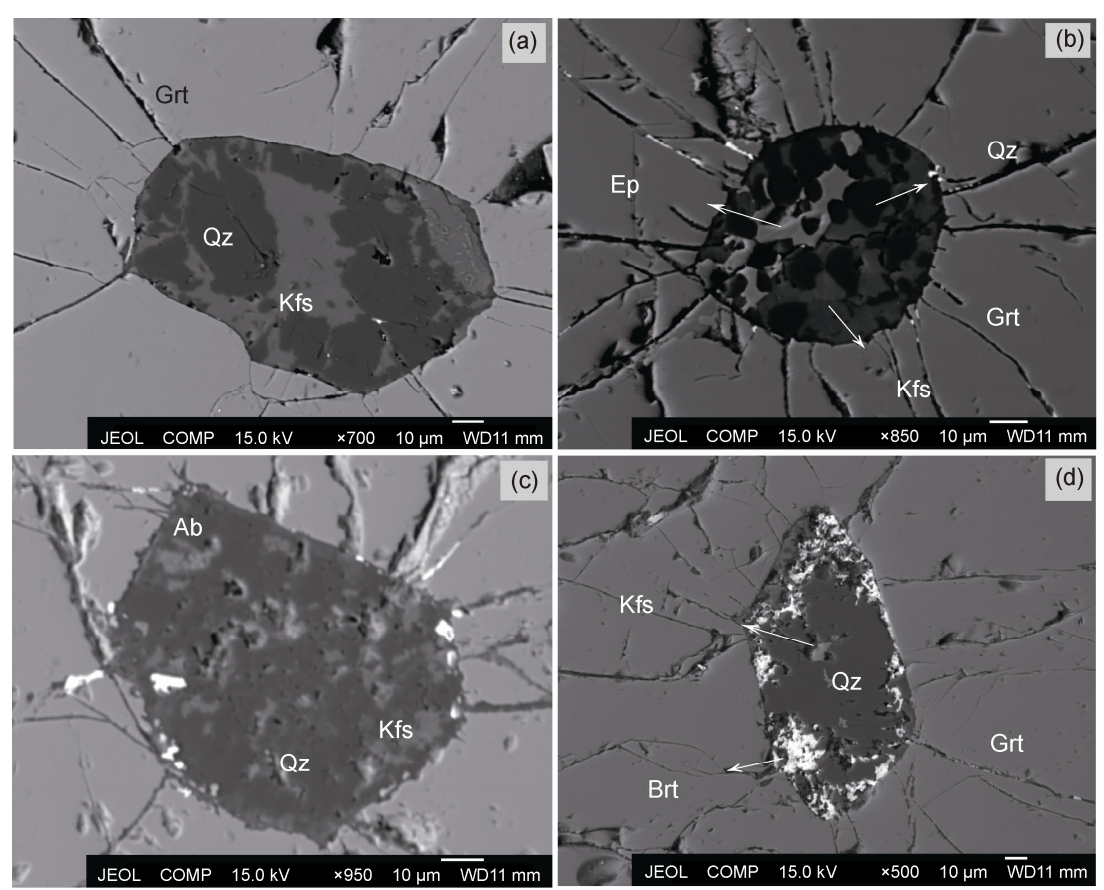

图 1 大别山超高压榴辉岩石榴石中的多相固体包裹体显微结构和矿物组成

引自文献[9,10]. (a) 钾长石+石英包裹体; (b) 钾长石+石英+绿帘石包裹体; (c) 钾长石+石英+钠长石包裹体; (d) 钾长石+石英+重晶石 包裹体. 矿物缩写: Grt, 石榴石; Kfs, 钾长石; Qz, 石英; Ep, 绿帘石; Ab, 钠长石; Brt, 重晶石 

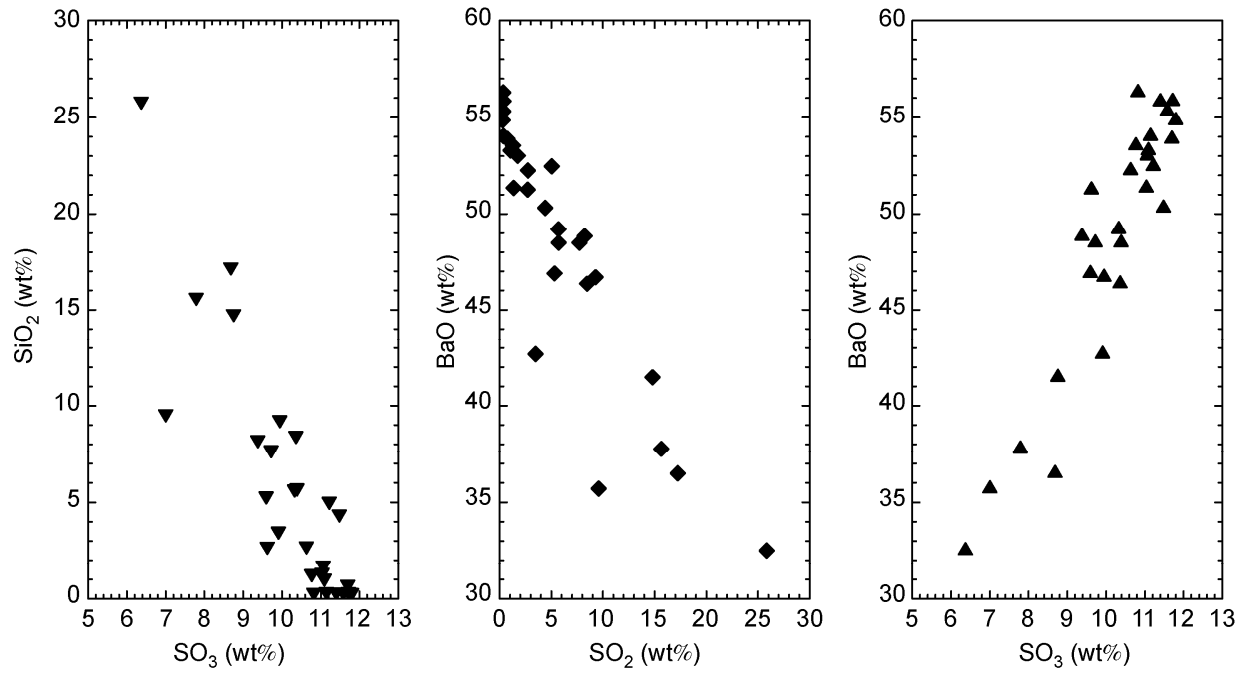

图 2 石榴石中包裹体重晶石 $\mathrm{SiO}_{2}, \mathrm{SO}_{3}$ 和 $\mathrm{BaO}$ 之间的相关性 修改自文献[9]

束斑大小, 这样对多相固体包裹体进行微量元素测 试时存在着较大的难度. 为了克服这个困难, 目前在 利用原位 LA-ICPMS 方法对这些多相固体包裹体进 行微量元素分析时, 分别采用外剥法和内剥法进行 分析. 外剥法是指采用较大的激光束斑, 在相同的分 析条件下，同时剥蚀包裹体及其周围的寄主矿物，然 后根据包裹体所占体积比, 对寄主矿物进行相应的 扣除, 得出包裹体的微量元素组成 ${ }^{[17 ~ 21]}$. 此方法虽 然简单易行, 但是对于包裹体与寄主矿物之间的分
布以及体积比的估计上存在着较大的误差和不确定 性. 内剥法是指选取半径相对较大的多相固体包裹 体, 用与之相近但偏小的激光束斑来剥蚀暴露到寄 主矿物表面的固体包裹体，此时所得的信号基本全 部来自包裹体.

对双河榴辉岩石榴石中长英质多相固体包裹体 (主要以 Kfs+Qz 为主)分别采用内剥法和外剥法进行 了微量元素测定 ${ }^{[10]}$. 结果显示, 两种方法给出了相 似的微量元素组成(图 3). 多相固体包裹体具有相对
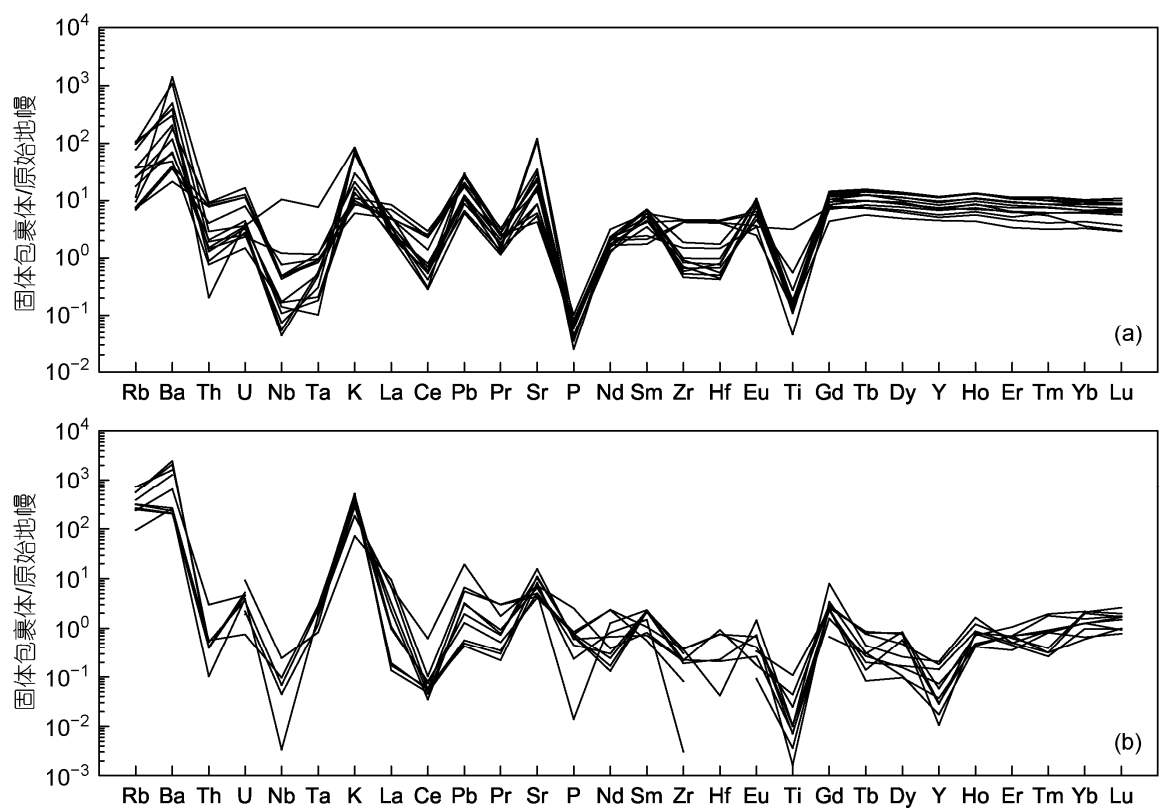

图 3 大别山榴辉岩中多相固体包裹体的微量元素分布蛛网图

数据引自文献[10]. (a) 外剥法; (b) 内剥法 
富集 LILE, $\mathrm{Sr}$ 和 $\mathrm{Pb}$, 但相对亏损 HFSE 的微量元素分 布型式, 与常见弧型岩浆岩的分布型式相近 ${ }^{[1]}$. 包裹 体表现出相对较高的 $\mathrm{K}, \mathrm{Rb}$ 和 $\mathrm{Ba}$ 含量, 可能与超高 压变质岩降压折返过程中多硅白云母脱水分解发生 部分熔融有关. 因此, 多相固体包裹体微量元素的分 析研究提供了超高压榴辉岩部分熔融产物的地球化 学组成.

\section{3 多相固体包裹体的形成机制}

大陆造山带超高压变质矿物中常含有大小不同 的各种固体包裹体, 它们有些属于显微出溶结构, 有 些具有交代成因, 有些则是矿物变质生长过程中捕 获的物质. 从成因分类来看, 固体包裹体包括受进/ 退变质作用的影响而发生反应后生成的残余矿物、偶 然捕获的矿物包裹体、矿物内部的出溶体、熔/流体 成因的多相固体包裹体等 ${ }^{[46,9]}$. 因此, 正确区分各种 类型的包裹体及其成因, 这是研究多相固体包裹体 需要解决的首要问题.

通过对大别造山带超高压变质岩中矿物进行多 相固体包裹体研究, 业已在变质矿物石榴石和绿辉石 中发现了不同类型的长英质多相固体包裹体 ${ }^{[5,6,8 \sim 10]}$. 结合前人对不同岩石体系中多硅白云母脱水熔融的 高温高压实验结果和双河榴辉岩形成的 $P-T$ 轨 迹 ${ }^{[13,16,22 ~ 26]}$, 可以估计出多硅白云母脱水熔融主要出 现在温度为 $830 \sim 750^{\circ} \mathrm{C}$ 、压力为 $2.4 \sim 1.6 \mathrm{GPa}$ 的一个 相对宽泛的 $P-T$ 范围内 (图 4 从 A D 点). 在这种条件 下, 脱水熔融出现在折返过程中从最大的峰期温度 到高压榴辉岩相阶段, 此时褐帘石、石榴石和金红石 仍处于相对稳定状态.

无论是锆石生长还是石榴石生长, 不仅需要变 质流体活动, 而且需要微量元素相对聚集. 因此, 超 高压变质岩中含水矿物的分解不仅是引起锆石和石 榴石生长的重要机制, 而且是引起超高压岩石部分 熔融的主要驱动力. 实验岩石学研究表明, 深俯冲大 陆地壳物质在超高压条件下可发生部分熔融 ${ }^{[27,28]}$. 在大别-苏鲁造山带超高压变质岩中的多相固体包裹 体是否是超高压熔体, 尚有待进一步研究. 由于多相 固体包裏体矿物中尚未发现深熔锆石, 因此没有对 大别造山带超高压变质岩发生部分熔融的时间进行 直接 U-Pb 定年. 不过, 先前的岩石学和地球化学研 究表明, 在深俯冲大陆地壳折返的早期, 超高压变质 岩发生过明显的降压脱水和部分熔融 ${ }^{[2,29]}$. 对双河榴

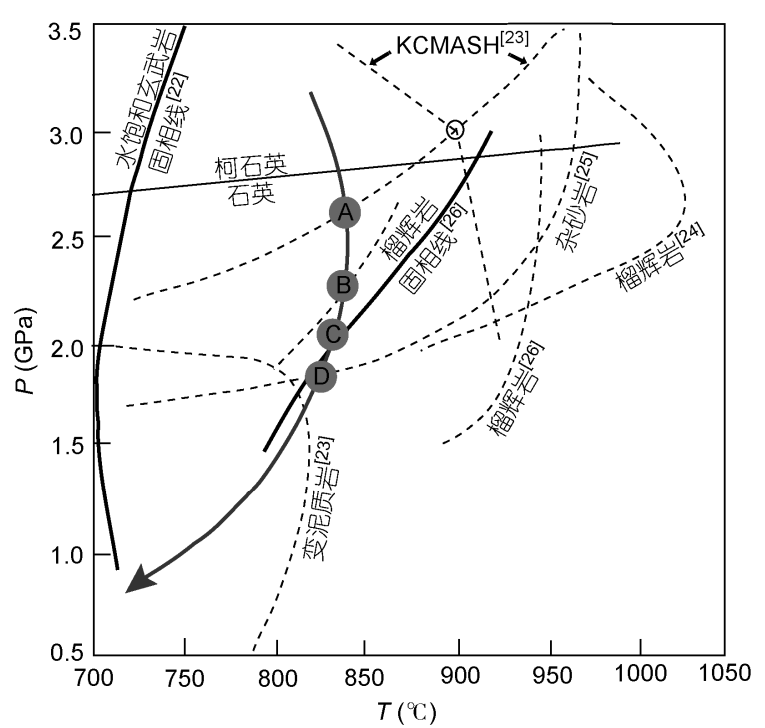

图 4 大陆俯冲带深部多硅白云母脱水引起榴辉岩部分熔融

修改自文献[9]. 虚线代表实验岩石学确定的多硅白云母脱水熔融 曲线(引自文献[22 26]), $P-T$ 轨迹上的 A D 点分别代表双河榴辉 岩在不同岩石体系下发生部分熔融的温压条件(详细解释参 见文献[9])

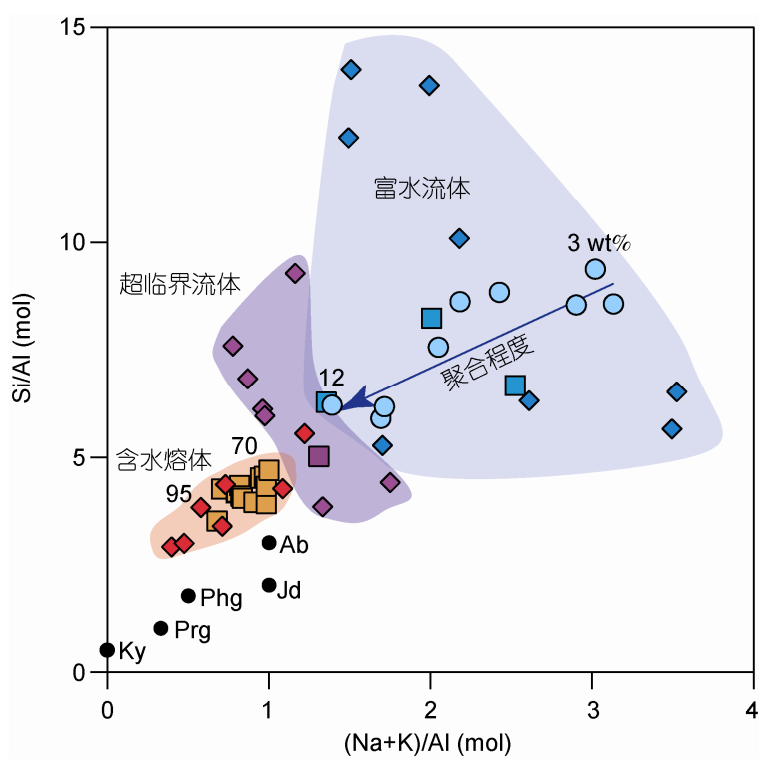

图 5 实验含水熔体和富水流体在主要元素组成上的差异

数据引自文献[34 36]. 数字代表流体中溶质含量百分数, 随熔体 聚合程度增高而增加. 矿物缩写: Ky, 蓝晶石; Prg, 钠云母; Phg, 多硅白云母; Jd, 硬玉; $A b$, 钠长石

辉岩中的锆石 U-Pb 定年和微量元素研究发现, 锆石 分别在 $244 \pm 3 \mathrm{Ma}$ 和 $225 \pm 2 \mathrm{Ma}$ 两个年龄发生幕式生 长 $^{[16,30]}$, 并且既有从变质流体中生长, 也有从深熔熔 
体中生长. 根据新生锆石中矿物包裹体和微量元素 组成, $\mathrm{Xia}$ 等人 ${ }^{[30]}$ 认为 $225 \pm 2 \mathrm{Ma}$ 生长的深熔锆石是超 高压岩石折返早期部分熔融的产物. 在苏鲁超高压带 中, 已有一系列研究来厘定宏观部分熔融现象及其产 物(淡色花岗岩脉或混合岩)的时代 ${ }^{[31,32]}$, 结果也是集 中在晚三叠世，对应于超高压岩石的折返阶段 ${ }^{[1]}$.

实验岩石学研究发现，在俯冲带高压-超高压变 质条件下存在 3 种不同类型的流体，即富水流体、含
水熔体和超临界流体 ${ }^{[2,33]}$. 综合应用各种岩石学和地 球化学方法分析矿物中的多相固体包裹体, 是认识 部分熔体性质的有效途径. 实验岩石学结果显示, 含 水熔体和富水流体在主要元素组成上存在显著差别 (图 5), 含水熔体的 $\mathrm{Si} / \mathrm{Al}$ 和 $(\mathrm{Na}+\mathrm{K}) / \mathrm{Al}$ 比值系统低于 富水流体，而超临界流体则呈现界于两者之间的过 渡特点. 对多相固体包裹体进行化学成分分析, 有可 能为识别大陆俯冲带深部熔/流体性质提供证据.

\section{参考文献}

1 Zheng Y F. Metamorphic chemical geodynamics in continental subduction zones. Chem Geol, 2012, 328: 5-48

2 Zheng Y F, Xia Q X, Chen R X, et al. Partial melting, fluid supercriticality and element mobility in ultrahigh-pressure metamorphic rocks during continental collision. Earth-Sci Rev, 2011, 107: 342-374

3 郑永飞. 超高压变质与大陆碰撞研究进展：以大别-苏鲁造山带为例. 科学通报, 2008, 53: 2129-2152

4 高晓英, 李姝宁, 郑永飞. 超高压变质矿物中的多相固体包裹体研究进展. 岩石学报, 2011, 27: 469-489

5 曾令森, 刘福来, 梁凤华, 等. 苏鲁榴辉岩钾长石+石英聚合体中重晶石矿物及其意义. 科学通报, 2007, 52: 1826-1840

6 曾令森, 梁凤华, Asimow P, 等. 深俯冲陆壳岩石部分熔融与苏鲁超高压榴辉岩中长英质多晶包裹体的形成. 科学通报, 2009, 54: $1826-1840$

7 曾令森, 高丽娥, 于俊杰, 等. 苏鲁仰口超高压岩石 SHRIMP 锆石 U/Pb 定年与部分熔融时限. 岩石学报, 2011, 27: 1085-1094

8 曾令森, 陈振宇, 陈晶. 苏鲁超高压榴辉岩长英质多晶包裹体中盐类固体包裹体. 科学通报, 2012, 57: 2972-2979

9 Gao X Y, Zheng Y F, Chen Y X. Dehydration melting of ultrahigh-pressure eclogite in the Dabie orogen: Evidence from multiphase solid inclusions in garnet. J Metamorph Geol, 2012, 30: 193-212

10 Gao X Y, Zheng Y F, Chen Y X, et al. Trace element composition of continentally subducted slab-derived melt: Insight from multiphase solid inclusions in ultrahigh-pressure eclogite in the Dabie orogen. J Metamorph Geol, 2013, 31: 453-468

11 Chen Y X, Zheng Y F, Hu Z C. Synexhumation anatexis of ultrahighpressure metamorphic rocks: Petrological evidence from granitic gneiss in the Sulu orogen. Lithos, 2013, 156-159: 69-96

12 Chen Y X, Zheng Y F, Hu Z. Petrological and zircon evidence for anatexis of UHP quartzite during continental collision in the Sulu orogen. J Metamorph Geol, 2013, 31: 389-413

13 Cong B L, Zhai M G, Carswell D A, et al. Petrogenesis of ultrahigh-pressure rocks and their country rocks at Shuanghe in the Dabie Mountains, Central China. Eur J Mineral, 1995, 7: 119-138

14 Zheng Y F, Fu B, Li Y L, et al. Oxygen and hydrogen isotope geochemistry of ultrahigh-pressure eclogites from the Dabie Mountains and Sulu terrane. Earth Planet Sci Lett, 1998, 155: 113-129

15 Li S G, Jagoutz E, Chen Y Z, et al. Sm-Nd and Rb-Sr isotopic chronology and cooling history of ultrahigh pressure metamorphic rocks and their country rocks at Shuanghe in the Dabie Mountains, Central China. Geochim Cosmochim Acta, 2000, 64: 1077-1093

16 Gao X Y, Zheng Y F, Chen Y X. U-Pb ages and trace elements in metamorphic zircon and titanite from UHP eclogite in the Dabie orogen: Constraints on $P$ - $T$ - $t$ path. J Metamorph Geol, 2011, 29: 721-740

17 Heinrich C A, Pettke T, Halter W E, et al. Quantitative multi-element analysis of minerals, fluid and melt inclusions by laser-ablation inductively-coupled-plasma mass spectrometry. Geochim Cosmochim Acta, 2003, 67: 3474-3497

18 Malaspina N, Hermann J, Scambelluri M, et al. Polyphase inclusions in garnet-orthopyroxenite (Dabie Shan, China) as monitors for metasomatism and fluid-related trace element transfer in subduction zone peridotite. Earth Planet Sci Lett, 2006, 249: 173-187

19 Malaspina N, Hermann J, Scambelluri M, et al. Multistage metasomatism in ultrahigh-pressure mafic rocks from the North Dabie Complex (China). Lithos, 2006, 90: 19-42

20 Malaspina N, Hermann J, Scambelluri M. Fluid/mineral interaction in UHP garnet peridotite. Lithos, 2009, 107: 38-52

21 Korsakov A V, Hermann J. Silicate and carbonate melt inclusions associated with diamonds in deeply subducted carbonate rocks. Earth Plane Sci Lett, 2006, 241: 104-118

22 Lambert I B, Wyllie P J. Melting of gabbro (quartz eclogite) with excess water to 35 kbar, with geological applications. J Geol, 1972, 80: $693-708$ 
23 Hermann J, Green D H. Experimental constraints on high pressure melting in subducted crust. Earth Planet Sci Lett, 2001, 188: 149-186

24 Skjerlie K P, Patino Douce A E. The fluid-absent partial melting of a zoisite-bearing quartz eclogite from 1.0 to 3.2 GPa: Implication for melting in thickened continental crust and for subduction-zone processes. J Petrol, 2002, 43: 291-314

25 Auzanneau E, Vielzeuf D, Schmidt M W. Experimental evidence of decompression melting during exhumation of subducted continental crust. Contrib Mineral Petrol, 2006, 152: 125-148

26 刘强，金振民，章军锋. 1.5 3.0 GPa 压力条件下多硅白云母榴辉岩的脱水熔融实验研究. 科学通报, 2009, 54: 1455-1464

27 Schmidt M W, Vielzeuf D, Auzanneau E. Melting and dissolution of subducting crust at high pressures: The key role of white mica. Earth Planet Sci Lett, 2004, 228: 65-84

28 Hermann J, Spandler C J. Sediment melts at sub-arc depths: An experimental study. J Petrol, 2008, 49: 717-740

29 Zheng Y F. Fluid regime in continental subduction zones: Petrological insights from ultrahigh-pressure metamorphic rocks. J Geol Soc, 2009, 166: 763-782

30 Xia Q X, Zheng Y F, Chen Y X. Protolith control on fluid availability for zircon growth during continental subduction-zone metamorphism in the Dabie orogen. J Asian Earth Sci, 2013, 67-68: 93-113

31 Liu F L, Robinson P T, Gerdes A, et al. Zircon U-Pb ages, REE concentrations and Hf isotope compositions of granitic leucosome and pegmatite from the north Sulu UHP terrane in China: Constraints on the timing and nature of partial melting. Lithos, 2010, 117: 247-268

32 Zong K Q, Liu Y S, Hu Z C, et al. Melting-induced fluid flow during exhumation of gneisses of the Sulu ultrahigh-pressure terrane. Lithos, 2010, 120: 490-510

33 Hermann J, Spandler C, Hack A, et al. Aqueous fluids and hydrous melts in high-pressure and ultra-high pressure rocks: Implications for element transfer in subduction zones. Lithos, 2006, 92: 399-417

34 Kessel R, Ulmer P, Pettke T, et al. The water-basalt system at 4 to $6 \mathrm{GPa}$ : Phase relations and second critical endpoint in a K-free eclogite at 700 to $1400^{\circ} \mathrm{C}$. Earth Planet Sci Lett, 2005, 273: 873-892

35 Hermann J, Spandler C. Sediment melts at sub-arc depths: An experimental study. J Petrol, 2008, 49: 717-740

36 Wohlers A, Manning C E, Thompson A B. Experimental investigation of the solubility of albite and jadeite in $\mathrm{H}_{2} \mathrm{O}$, with paragonite + quartz at 500 and $600^{\circ} \mathrm{C}$, and 1-2.25 GPa. Geochim Cosmochim Acta, 2011, 75: 2924-2939 